\title{
A Review of Agility: Practical Applications for Strength and Conditioning
}

Warren Young, PhD

University of Ballarat, Ballarat, Australia

Damian Farrow, PhD

Australian Institute of Sport, Belconnen, Australia

\section{s u m m a r y}

Agility is an important component of many sports but has not been extensively researched. The various components that contribute to agility performance are discussed and training guidelines are provided. There appears to be limited transfer to agility performance from straight sprint training as well as from general strength training. The principle of training specificity is emphasized to achieve maximum transfer to onfield performance.

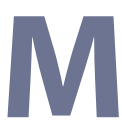
any sports performed on a court or on a field require high-speed total body movements. Many of these are in response to the motion of a ball, opposition players, or teammates. This important component of athletic performance may be described as agility, and it is sometimes grouped together with terms such as speed and quickness. The development of agility is often the responsibility of the strength and conditioning coach, but a clear understanding of the characteristics of agile performers and the best way to train agility appears to be elusive, especially compared to other components of physical preparation such as explosive power. Therefore, the purpose of this article is to review what is known about agility and to provide guidelines for the development of this important quality.

Agility is a complex quality and in recognizing this, it has been stated that

...agility permits an athlete to react to a stimulus, start quickly and efficiently, move in the correct direction, and be ready to change direction or stop quickly to make a play in a fast, smooth, efficient, and repeatable manner (20, pp. 140-141).

The multifactorial nature of agility has also been represented by a deterministic model indicating the various factors contributing to performance (22) (Figure 1). The following discussion is intended to reveal the applications that follow from the available research evidence. Identifying the relative importance of the various contributing factors may assist coaches in developing training strategies to maximize the development of agility. Figure 1 will be used to guide this analysis.

In baseball or softball, a batter produces a rapid change of direction (COD) to run around the bases. Although this has traditionally been described as agility, it is a somewhat rare situation because the COD movement is preplanned. In most sports, such as any football code or basketball, a COD is produced in response to a stimulus such as an opponent's actions. Therefore, the speed of this movement is influenced by perceptual and decision-making factors, such as the speed of anticipation of the player. For this reason, the ability to change direction and velocity in a preplanned movement, such as in base running, may be better described as COD speed.

\section{Technique}

The ability to change direction and velocity quickly would be expected to be influenced by the body position adopted while running. A forward lean is required to accelerate, a backward lean to decelerate and stop, and a sideward lean to produce a lateral COD. These body positions are necessary in order to produce forces to the ground to evoke reac- 


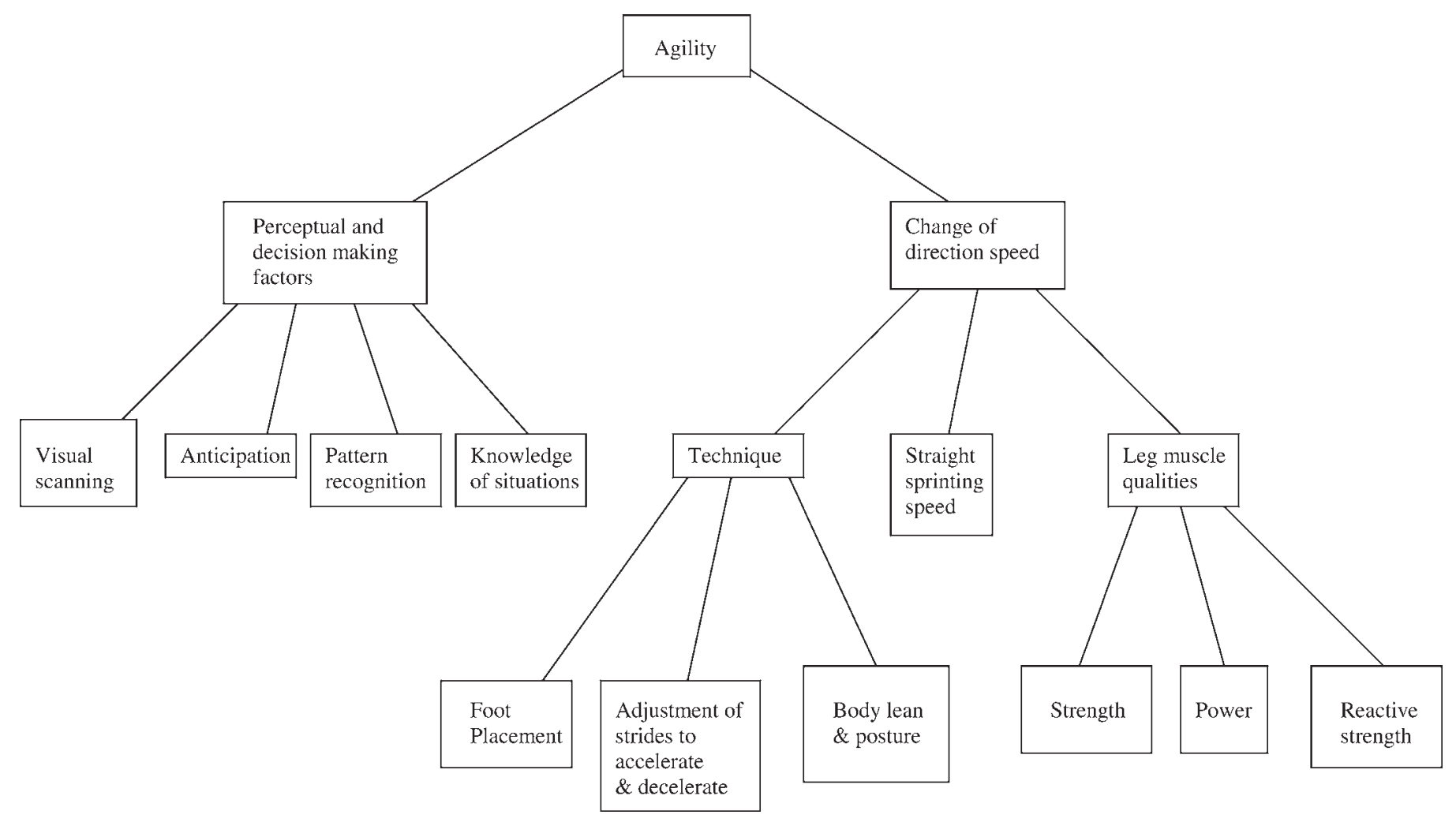

Figure 1. Deterministic model of agility performance (22).

tion forces in the desired direction. For example, a player must lean to the left and plant the right foot to the right of the body to push into the ground in that direction. The ground reaction force will be directed to the left and will produce the necessary change in direction (Figure 2). If an athlete is running in a straight line with classical running posture as demonstrated by a 100 -m sprinter, he or she will need to make fast adjustments to the running posture to execute a fast COD. Such a fast adjustment of posture and positioning of limbs is clearly a skill that would be expected to be trainable.

Some authors have discussed technical aspects of agility. For example, Sayers (17) qualitatively assessed the running techniques used by rugby players during games and concluded that they used a modified technique that was better suited to producing a fast COD compared to the technique exhibited by track ath- letes. The rugby players were said to run lower and use a relatively high step frequency compared to track sprinters. Brown and Vescovi (4) proposed training drills to develop an arm action conducive to fast COD movements. Although suggestions relating to the technique used to change direction quickly can be found in the coaching literature, we could not find any biomechanical research relating to the optimum technique for maximizing COD speed.

\section{Straight Sprinting Speed}

Are the fastest sprinters also the most agile athletes? Are the sprint training methods used by track and field coaches effective for the development of COD speed? One way to investigate the relationship between straight sprinting speed and COD speed is to determine the correlation between the 2 qualities. When the correlation coefficient is $<0.71$, the common variance between the 2 tests is less than $50 \%$, and the qual- ities measured can be said to be specific in nature (18). Using such a statistical analysis, there are many research reports indicating specificity between tests of straight speed and COD speed $(7,8,14)$. It could be argued that the relatively low correlation reported in these studies was because of the fact that the total distances covered in the straight and COD speed tests were different. However, studies using the same running distances have also reported a specificity of straight and COD speed tests $(5,21,24)$.

Perhaps a more important question is, does straight sprint training transfer to COD speed? Such a question was addressed in a training study by Young et al. (24). Participants performed either straight sprint training or COD sprints involving 3-5 changes of direction in a zigzag pattern over a 6 -week period. It was found that the straight sprint training enhanced straight speed significantly by approximately $3 \%$, but produced 


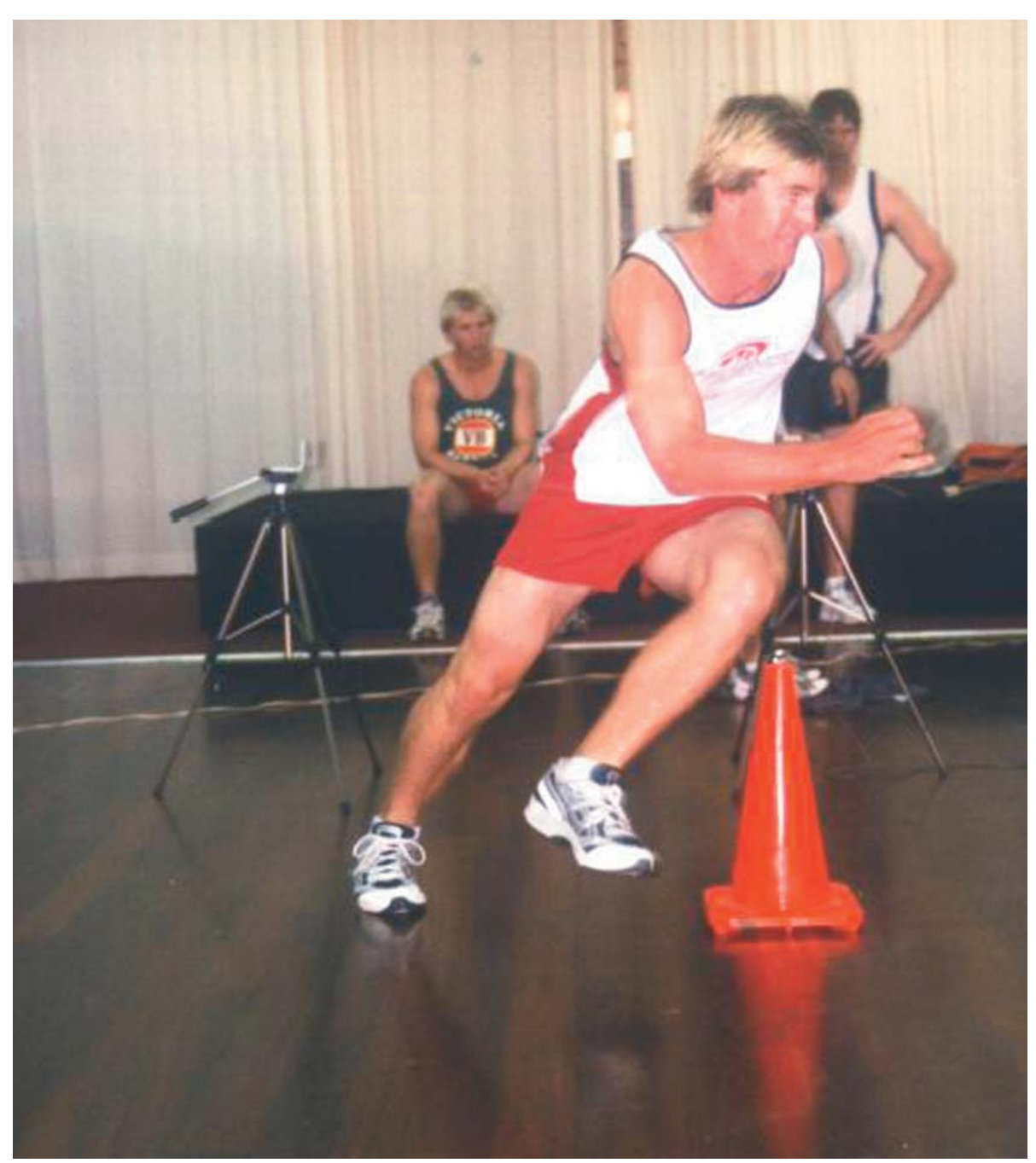

Figure 2. Lateral change of direction.

no gain in a test containing 5 changes of direction. Conversely, the COD speed training had limited transfer to straight sprinting speed. These findings can be explained by training specificity, and suggest that athletes must train specifically with the movement patterns required in their sports to benefit optimally from their training.

\section{Leg Muscle Qualities}

It is an oversimplification to suggest that the leg muscle groups are solely responsible for COD movements. For example, a rapid extension of the leg might produce high forces laterally to the ground, but the ground reaction forces will not be effective for propulsion of the center of between maximum leg strength and COD speed is lacking. In contrast, there have been many studies relating various measures of leg muscle power to some test of COD speed. This research has consistently found low-to-moderate correlations, indicating a specificity of these qualities $(9,14,16,21,22)$.

Reactive strength has been defined as the ability to quickly change from the eccentric to the concentric phase in a stretchshortening cycle (SSC) muscle contraction sequence, and is a relatively specific form of muscle power (23). A cutting movement to produce a lateral change in direction typically involves such a SSC muscle sequence. Therefore, it might be expected that tests of reactive strength would correlate highly with lateral COD speed. However, using various drop jump (DJ) tests as measures of reactive strength, research has found relationships with various COD speed tests to be moderate, with common variances of less than $50 \%(9,21,22)$. One study (22) used a single-leg DJ test to identify leg muscle imbalances in reactive strength. This study also noted differences in COD speed in producing a single turn to the left and to the right. Although the correlation between reactive strength and COD speed wasn't particularly high, it was noted that the subjects possessed significantly greater reactive strength in the right leg and produced significantly better COD speed to the left side. Although the reactive strength imbalance between the right and left sides could have been responsible for the COD speed imbalance, these data do not prove a cause-and-effect relationship.

McBride et al. (15) conducted a training study involving 8 weeks of jump squat training in a Smith machine. Training with $30 \%$ of 1 repetition maximum (1RM) induced significant gains in 1RM squat strength (8\%) and jumping power with a load of $30 \%$ of $1 \mathrm{RM}$ (10\%), and was accompanied by smaller $(1.7 \%)$ but significant gains in COD speed, as measured by the T-test. Hoff- 
man et al. (13) compared training with powerlifting and weightlifting exercises in American football players. Both training protocols were able to produce significant gains in 1RM squat strength of approximately $13 \%$, but there were no significant increases in vertical jumping power, straight sprinting speed, or COD speed measured by the T-test. An 8 -week training study evaluated the effects of a program using weightlifting exercises and a program containing plyometric exercises (19). Both training methods were successful in achieving significant improvements in squat 1RM and vertical jumping height, but neither was able to significantly improve $30-\mathrm{m}$ sprint time or COD speed, as measured by a test requiring 11 changes of direction. Interestingly, some of the plyometric exercises involved single-leg takeoffs, but none used lateral movements. A plyometric training program involving jumping exercises that contain singleleg lateral takeoffs, e.g., bounding in a zigzag pattern, might be expected to achieve better transfer to COD speed. Nevertheless, these training studies indicate that various resistance training modalities that can produce gains in strength and power produce much more modest changes in COD speed tests.

\section{Perception and Decision Making}

Most of the coaching and research literature pertaining to agility refers to either training or testing that involves preplanned changes of direction. This is typically achieved by using such obstacles as cones, poles, or ladders. It is not difficult to identify an athlete who is rated as outstanding in on-field agility but considered to possess average athleticism based on fitness testing. Such an athlete may have average COD speed but be very agile because he or she is highly skilled in the perceptual and decision-making factors (Figure 1).

Occasionally tests have been devised that have required subjects to change direction in response to a stimulus such as a light $(3,6,12)$. These tests incorporate reaction time as a component of the total time taken from the onset of a stimulus to the completion of an agility task. As such, an athlete with a shorter reaction time may have an advantage in agility performance. However, the speed at which a response is made on field is not determined only by simple reaction time. Many athletes will anticipate what is about to occur on the field and the direction in which an opponent is about to move.

There is considerable research indicating that better athletes produce more accurate and faster responses because of their capacity to pick up anticipatory information (2). In situations demanding agility, a primary information source is the posture or kinematics of an opponent. For example, in football, perceptually-skilled players use cues coming from the angle of the hips to anticipate the direction of an opponent's dodge. Lesser-skilled players, however, are not necessarily attuned to the same information. This tight coupling between the kinematics and movement probabilities of an opponent questions the value of using nonsport-specific training stimuli such as lights and auditory cues. The perceptual-motor literature strongly suggests that unless the stimulus is sport-specific, skilled athletes are unable to use their perceptual skill to advantage (1).

Recently an agility test was devised that required netball players to respond to a life-size video clip of an attacking player about to pass a ball (11). The subject was required to shuffle to the side, move forward, and then break to the left or right as soon as she decided in which direction the ball would be passed. With the aid of video analysis, decision time was recorded as the time elapsed from ball release to the instant the foot was planted to change direction. It was found that more-highly-skilled players were faster in total movement time in this reactive agility task, and this was partly because of faster decision times. The less-skilled players initiated their COD movement after ball release, whereas the moreskilled players moved before ball release, suggesting that the better players anticipated the pass direction.

Besier et al. (3) conducted a biomechanical analysis on planned and unplanned cutting movements. The unplanned movements were produced after responding to a light stimulus. Although this was not a sport-specific stimulus, it did impose a time constraint on the subjects. The authors reported that there were significantly greater loads on the knee joint in the unplanned cutting task, which would increase the risk of knee ligament injury. They suggested that this may have been because of insufficient time to make the necessary postural adjustments and place the leg in an optimum position. Therefore, learning to respond more quickly to a COD stimulus in sport may not only enhance performance but also reduce the risk of injury.

\section{Conclusions and Practical Applications}

Although there is much research that has used various agility tests as part of a test battery, there is limited research that has focused specifically on the enhancement of agility performance. Further, there is no well-accepted definition of agility, and there are a multitude of tests that have been used. A difficulty with these tests is that they may contain a variety of movement patterns, such as forward sprinting, backwards running, sideways shuffling, lateral cutting, and lateral crossover stepping. The number of directional changes and the angle of change vary considerably among the tests. Therefore, it is impossible to isolate what components of agility are most important. Perhaps this is why some strength and conditioning coaches seem to favor a general training approach to agility development, utilizing as many movement patterns as possible. 


\section{Technique}

Although some coaches attempt to teach agility skills, there is no research to support an optimum technical model of how an athlete should adjust the body position and limbs to change direction. This is in marked contrast to the enormous quantity of research concerning sprinting in a straight line, which is a predictable closed skill. With this in mind, 2 guidelines can be offered. The first is to identify the specific movement patterns used by successful athletes in a particular sport. The practice of these movements would be expected to yield better transfer to on-field performance than a generalized approach. The other guideline is to practice agility skills under the time constraints of game situations, because this forces the athlete to make fast postural adjustments to the demands of the situation. Practice of COD skills using obstacles to direct movement patterns is limited in that it allows postural adjustments to be preplanned without time constraints, and also may produce unnatural movements, movements that do not occur in a game.

\section{Sprint Training}

Research supports the notion that sprinting in a straight line is clearly a different skill from agility movement patterns. Because of the principle of specificity, straight-line sprinting speed cannot be expected to transfer to agility. If a sport involves straight sprinting as well as agility maneuvers, conventional sprint training can be justified. As with sprint training, agility training sessions should be structured to match the energy system requirements of the sport. For example, some agility training should be performed at maximum intensity with complete rests between efforts, whereas short recoveries might be prescribed to target the development of agility-endurance.

\section{Strength Training}

The available research provides little support that leg muscle strength, power, and reactive strength are major contributors to agility performance. However, a rationale was made to suggest that plyo- metric training that involves single-leg lateral movements could potentially be beneficial to COD speed development. It is likely that as agility skills become more complex with more and sharper changes of direction, leg muscle qualities have a diminishing contribution. However, some general strength training for core stability and balance can still be recommended to develop the basic movement efficiency of the athlete.

\section{Perception and Decision Making}

Based on the differences in the way a skilled decision-maker uses anticipatory information relative to less-skilled athletes, the training applications are obvious. Activities that force an athlete to pay attention to the specific movement kinematics of the opponent are vital. For example, tag games become a valuable means of improving agility in team sports. Importantly, methods that force the player to make a choice in response to the early appearance of such kinematic information have been demonstrated as a successful method of training anticipation (10). In these methods, known as temporal occlusion training, players are forced to learn the link between an early cue and its resultant outcome. The use of edited video footage filmed from the perspective of the reacting player is a popular example of this training approach. For example, a player may be required to watch an opponent attempt to dodge in front of him; however, the vision is occluded a step before the dodging player initiates his COD and the player is required to react on the basis of the information available.

\section{References}

1. Abernethy, B., K.T. Thomas, And J.T. THOMAS. Strategies for improving understanding of motor expertise (or mistakes we have made and things we have learned!!). In: Cognitive Issues in Motor Expertise. J.L. Starkes and F. Allard, eds. Amsterdam, The Netherlands: Elsevier Science Publishers, B.V., 1993. pp. 317-356.
2. Abernethy, B., J. Wann, and S. PARKS. Training perceptual motor skills for sport. In: Training for Sport: Applying Sport Science. B. Elliott, ed., Chichester: John Wiley, 1998. pp. $1-68$.

3. Besier, T.F., D.G. Lloyd, J.L. Cochrane, and T.R. Ackland. External loading of the knee joint during running and cutting maneuvers. Med. Sci. Sports Exerc. 33:1168-1175. 2001.

4. Brown, T.D., AND J.D. Vescovi. Efficient arms for efficient agility. Strength Cond. J. 25(4):7-11. 2003.

5. Buttifant, D., K. Graham, and K. Cross. Agility and speed in soccer players are two different performance parameters. In: Science and Football IV. W. Spinks, ed. London: Routledge, 2002, pp. 329-332.

6. Chelladurai, P., M.S. Yuhasz, and R. Sipura, The reactive agility test. Percept. Mot. Skills. 44:1319-1324. 1977.

7. Draper, J.A., and M.G. Lancaster. The 505 test: A test for agility in the horizontal plane. Aust. J. Sci. Med. Sport. 17, 1:15-18. 1985.

8. Draper, J., and F. Pyke. Turning speed: A valuable asset in cricket run making. Sports Coach. 11(3):30-31. 1988.

9. Djekalikian, R. The relationship between asymmetrical leg power and change of running direction. Master's thesis, University of North Carolina, Eugene, OR: Microform Publications, University of Oregon. 1993.

10. Farrow, D., and B. Abernethy. Can anticipatory skills be learned through implicit video-based perceptual training? J. Sports Sci. 20:471-485. 2002.

11. Farrow, D., W. Young, and L. BRUCE. The development of a test of reactive agility for netball: A new methodology. J. Sci. Med. Sport. 8(1):52-60. 2005.

12. Hertel, J., C.J. Denegar, P.D. JohnSON, S.A. Hale, and W.E. BuCKLey. Reliability of the Cybex reactor in the assessment of an agility task. J. Sport Rehab. 8:24-31. 1999.

13. Hoffman, J.R., J. CoOper, M. Wen- 
DELL, AND J. Kang. Comparison of Olympic vs. traditional power lifting training programs in football players. $J$ Strength Cond. Res. 18(1):129-135. 2004.

14. Mayhew, J.L, F.C. Piper, T.M. SChwegler, and T.E. Ball. Contributions of speed, agility and body composition to anaerobic power measurements in college football players. J. Appl. Sports Sci. Res. 3(4):101-106. 1989.

15. McBride, J.M., T. TriplettMcBride, A. Davie, AND R.U. NewTON. The effect of heavy- vs. light-load jump squats on the development of strength, power, and speed. J. Strength Cond. Res. 16(1):75-82. 2002.

16. Negrete, R., AND J. Brophy. The relationship between isokinetic open and closed chain lower extremity strength and functional performance. J. Sport Rehabil. 9:46-61. 2000.

17. SAYERS, M. Running techniques for field sport players. Sports Coach. 23(1):26-27. 2000.

18. Thomas, J.R., and J.K. Nelson. Research Methods in Physical Activity. Champaign, IL: Human Kinetics, 1990.

19. Tricoli, V., L. Lamas, R. Carnevale, AND C. UGRINOwitsCh. Short-term effects on lower-body functional power development: Weightlifting vs. vertical jump training programs. J. Strength Cond. Res. 19(2): 433-437.2005.

20. Verstegen, M., and B. Marcello. Agility and coordination. In: High Performance Sports Conditioning. B. Foran, ed. Champaign, IL: Human Kinetics, 2001. pp. 139-165.

21. Young, W., M. Hawken, And L. McDONALD. Relationship between speed, agility and strength qualities in Australian rules football. Strength Cond. Coach. 4(4):3-6. 1996.

22. Young, W., R. JAMES, AND I. MontGOMERY. Is muscle power related to running speed with changes of direction? J. Sports Med. Phys. Fitness. 42:282-288. 2002.

23. Young., W., G. Wilson, and C. BYRNE. Relationship between strength qualities and performance in standing and run-up vertical jumps. J. Sports Med. Phys. Fitness. 39:285-293. 1999.

24. Young., W.B., M.H. McDowell, and B.J. Scarlett. Specificity of sprint and agility training methods. J. Strength Cond. Res.. 15(3):315-319. 2001.

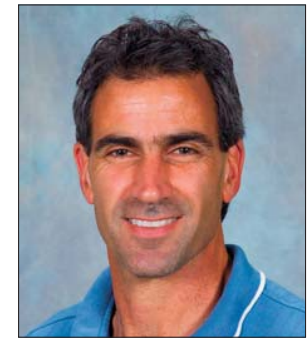

Young

Warren Young is a Senior Lecturer at the University of Ballarat, Ballarat, Victoria, Australia.

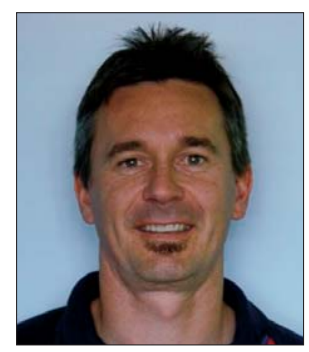

Farrow

Damian Farrow is the Skill Acquisition Specialist at the Australian Institute of Sport, Canberra, Australia. 